\title{
Cellulose Monolith with Tunable Functionality for Immobilization of Influenza Virus
}

\author{
Xinghua Pan ${ }^{1}$, Sudheer Jinka ${ }^{2}$, Vinit Kumar Singh ${ }^{2}$, Seshadri Ramkumar ${ }^{2}$ and M Nazmul Karim ${ }^{1^{*}}$ \\ ${ }^{1}$ Artie McFerrin Department of Chemical Engineering, Texas A and M University, Texas, USA \\ ${ }^{2}$ Nonwovens and Advanced Materials Laboratory, Texas Tech University, Texas, USA
}

"Corresponding author: M Nazmul Karim, Artie McFerrin Department of Chemical Engineering, Texas A and M University, College Station, TX 77843 , USA, Tel: +1979 845 9806; Fax: +1 979845 3266; E-mail: naz.karim@che.tamu.edu

Received Date: March 20, 2018; Accepted Date: April 6, 2018; Published Date: April 12, 2018

Copyright: () 2018 Pan X, et al. This is an open-access article distributed under the terms of the Creative Commons Attribution License, which permits unrestricted use, distribution, and reproduction in any medium, provided the original author and source are credited.

\begin{abstract}
Advancements in influenza virus or virus like particle separation technology can greatly accelerate vaccine production. Due to frequent mutation of the influenza virus, rapid and effective separation of influenza virus directly from host cells still remains a challenge. In this paper, we developed a new cellulose fiber monolith-based chromatography with tunable anion exchange and pseudo-affinity modifications for the separation of cell cultureproduced influenza virus. A novel strategy is proposed for preparing cellulose fiber monolith, which enhances the mechanical property of the monolith. Controllable pore size and porosity were achieved by dispersing and removing a porogen into cellulose-dissolving solution. The structure of cellulose monolith was characterized by Scanning Electron Microscope and the porosity study, which confirmed the controllable average pore size of 10 micron and porosity up to $85 \%$. Two virus separation modes including anion-exchange and pseudo-affinity were introduced into the cellulose monolith, subsequently. The two modifications were quantified by an Element Analyzer. A H1N1 influenza virus strain (A/WSN/33) was produced and harvested in adherent Madin-Darby Canine Kidney Epithelial (MDCK) cells and used directly for virus separation. The results indicated that the pseudo-affinity mode had higher virus separation efficiency and had less host cell dsDNA and protein contamination.
\end{abstract}

Keywords: Cellulose monolith; Influenza virus separation; Pseudoaffinity

\section{Introduction}

The influenza virus undergoes frequent mutations which makes vaccine immunoprophylaxis (currently, the most efficient method for flu prevention) a challenge [1]. Efficiency of vaccine production has been greatly improved by using Escherichia coli (E. coli) or a mammalian cell line as the virus-producing host, instead of conventional chicken eggs. Nevertheless, purification of the virus or virus like particle (VLP) from host cells remains a time consuming procedure and is still undergoing extensive study [2-4].

The separation of virus or virus like particle (VLP) involves three basic steps: clarification, concentration, and polishing. Filtration, centrifuge, and liquid chromatography are widely used in these processes based on the physicochemical properties of the virus, such as size, density, and surface charge $[2,5,6]$. Filtration and centrifugation are primarily used to remove host cell debris and precipitates. Liquid chromatography techniques such as affinity, size exclusion and ion exchange are used to isolate the virus or VLP. Generally, affinity chromatography has the highest specificity to the surface protein of virus but suffers from high cost and ligand instability [7]. Ion-exchange mode can be easily introduced but it is more susceptible to contamination of the host cell DNA and proteins due to the nonspecific electrostatic interaction. Higher separation efficiency could be achieved by sequentially combining various separation methods.

Monoliths are single-piece interconnected porous stationary phase material, which are applicable for separation technology such as gas and liquid chromatography, high performance liquid chromatography, and capillary electro chromatography [8-10]. The separation targets cover from small molecules such as gas to large complex biomolecules. The advantages of monoliths compared to the traditional porous beads are their mechanical robustness, low cost and ease of in situ preparation, no void volume, controllable pore size, and easy functionalization to suit application in ion-exchange, affinity, chiral, and mixed operations [11-13]. Another advantage of monoliths is that they can be easily shaped into various modes such as capillary, column, micropipette tips, and microfluidic channel 12. Besides, highly interconnected porous structure allows high hydraulic permeability and convective flow dynamics in monolith, which allows higher flux rate. The applications of virus separation through monolith have been demonstrated [14-16].

Currently, two types of monolith have been widely studied: silica monolith and organic polymer monolith. Preparation of silica monolith has been extensively reported with controlled shape and pore size from mesoporous to macroporous $[17,18]$. The organic polymeric monoliths are prepared from nearly thirty diverse polymers, which can be primarily categorized into methacrylate and acrylate based polymer groups [11,19]. The structure of organic polymer monolith was controlled by porogen and crosslinking agents.

Cellulose is one of the promising alternatives for the methacrylate and silica monolith due to its excellent biodegradability, sustainability, and biocompatibility which make it an ideal candidate for medical and biochemical applications. The organic dissolving solution used for processing cellulose could be recycled which makes the whole process environment-friendly. Numerous hydroxyl groups on cellulose allow for functionalization for different applications. Cellulose-based material has been widely applied in separation technology such as in centrifuge and filtration membrane, column matrix in ion-exchange 
chromatography, and affinity chromatography [20]. Besides, cellulose membranes have low non-specific interaction with proteins, making it a suitable separation matrix for biomolecules [21,22].

The preparation of cellulose monolith has not been exclusively reported yet, especially regarding its preparation process, mechanical properties, and structural property. In this paper, a method was developed to prepare cellulose fiber monolith with two tunable modes of functionalization for separating the influenza virus directly from mammalian cell culture media.

Novel cellulose monolith was prepared through the "partially dissolving-reshaping" process of cellulose fiber, using ethylendiamine (ED) and potassium thiocyanate (KSCN) as co-dissolving solution, and the pore size and porosity of the monolith were controlled by a porogen, calcium carbonate, as shown in Figure 1.

The cellulose monolith was functionalized inside the interconnected structure for virus separation. Two modes of functionalization were introduced sequentially. Anion-exchange functionalization was first introduced into the monolith matrix by mixing the anion-modified cellulose with the Lyocell cellulose fiber. A second modification, pseudo-affinity, was introduced into the cellulose monolith by selfassembling sulfated cellulose fiber onto the outer and inner surface of the monolith.

\section{Experimental Section}

\section{Materials}

Ethylendiamine (ED) and potassium thiocyanate (KSCN) were purchased from Sigma-Aldrich, USA. Lyocell cellulose fiber (1.7 dtex, $51 \mathrm{~mm}$ nondull) was provided by Lenzing Corporation, USA. Diethylaminoethyl (DEAE)-cellulose was bought from Sigma-Aldrich, USA. Calcium carbonate with diameter of $10 \mu \mathrm{m}$ and $3 \mu \mathrm{m}$ was obtained from Specialty Minerals Inc., USA. Adherent Madin-Darby Canine Kidney (MDCK) cells and A/WSN/33 (WSN, H1N1) virus were provided by Health Sciences Center at Texas Tech University. MDCK cells were infected by WSN virus in Dulbecco Modified Eagle Medium (DMEM). Then, the tissue-culture-infective doses (TCID50) of WSN influenza virus were measured using a 96-well device before and after the cellulose fiber monolith separation.

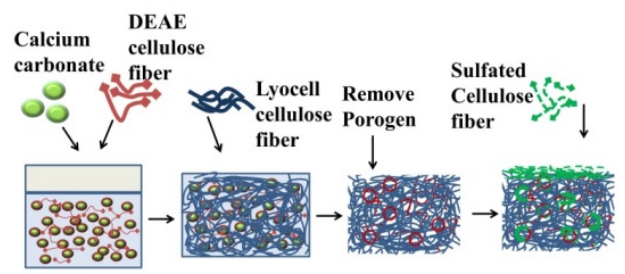

Figure 1: Illustration of cellulose fiber monolith preparation process.

\section{Methods}

Sulfate modification of cellulose fiber: Sulfate modification of cellulose fiber was performed according to the reference $[23,24]$. In brief, sulfuric acid was dropwise added to the pre-cooled ethanol, and cellulose fiber was added into the solution then stirred for 4.5 hours at $0^{\circ} \mathrm{C}$. Then the cellulose fiber was washed repeatedly by pre-cooled acetone. Sulfated cellulose fiber was dried and suspended in water using sodium hydroxide to adjust the $\mathrm{pH}$ to 7.5.

Preparation of cellulose fiber monolith: Cellulose fiber monolith was prepared by a partially dissolving-reshaping method. Briefly, $7.8 \mathrm{~g}$ of KSCN was dissolved in $16.6 \mathrm{ml}$ of ED solution under stirring. A sample of $0.03 \mathrm{~g}$ of DEAE-cellulose fiber was dispersed together with $15.5 \mathrm{~g}$ of calcium carbonate in the above-mentioned solution under vigorous stirring at $500 \mathrm{rpm}$. Then $0.8 \mathrm{~g}$ of DEAE cellulose and $0.8 \mathrm{~g}$ of cellulose Lyocell fiber was mixed with the dispersion subsequently, and transferred into a cylindrical mold for shaping. The monolith was kept at $-20^{\circ} \mathrm{C}$ before being transferred into acetone solution for solvent exchange. The monolith was immersed in acetone for $48 \mathrm{~h}$, then transferred to water for solution exchange for another 48 hours under continuously stirring. Porous structure was generated by dissolving calcium carbonate using hydroxyl chloride solution $(0.35 \% \mathrm{w} / \mathrm{v})$. The monolith was treated with hydroxyl chloride solution under stirring until calcium carbonate was totally dissolved and removed. The monolith was then washed with DI water under stirring.

Functionalization of pseudo-affinity mode on monolith: The pseudo-affinity (sulfated cellulose) functionalization was introduced into the monolith by continuous flowing of the sulfated cellulose fiber suspension $(3.5 \% \mathrm{w} / \mathrm{v})$ through the monolith at a speed of $0.02 \mathrm{ml} / \mathrm{min}$ with a total volume of $30 \mathrm{ml}$. Then, the monolith was washed by flow buffer (10 mM Tris-HCl, $150 \mathrm{mM} \mathrm{NaCl}, \mathrm{pH}$ 7.8.).

Separation of influenza virus: Influenza virus was separated using both anion-exchange and pseudo-affinity monolith in an ÄKTA FPLC (GE, US) system. Influenza virus A/WSN/33 (WSN, H1N1) produced in MDCK cells was harvested and frozen at $-80^{\circ} \mathrm{C}$ according to the established protocol [25]. Cellulose monolith was cut into sections with a height of $1 \mathrm{~cm}$ and fit into a superloop (1/16" fittings). Influenza virus-containing suspension $(1 \mathrm{~mL})$ was thawed and flowed through monolith separation directly without any treatment. The flow rate was controlled at $0.5 \mathrm{~mL} / \mathrm{min}$. Separation was monitored by a UV system. Flow buffer has $10 \mathrm{mM}$ of Tris and $150 \mathrm{mM}$ of $\mathrm{NaCl}$, with $\mathrm{pH}$ at 7.8. Virus concentration was determined by TCID50 before and after the separation. The concentrations of dsDNA and protein were measured using Quant-iTM Pico-Green dsDNA reagent from Invitrogen and BioRad Assay, respectively.

Mechanical property determination: Compression test has been conducted using Instron 5569 Universal Tester with a $500 \mathrm{~N}$ load cell and equipped with two flat surface compression stages. Cellulose samples were cut into $20 \mathrm{~mm}$ diameter cylinders with a height of 10-12 $\mathrm{mm}$. The top stage was first set to move downwards at a rate of 10 $\mathrm{mm} / \mathrm{min}$ and compression stress and compression strain data was obtained. Two different sets of measurements were performed. First, three different samples were tested at $30 \%$ compression. The second experiment was to set compression strain at $30 \%, 40 \%$, and $50 \%$, individually. All the samples used in the mechanical property measurement were prepared using the same procedure, and fiber content refers to the fiber proportion at the beginning of the process.

Porous structure determination: pore size and structure: Microscopic structure of cellulose monolith was observed by Hitachi 
S-4700 field emission Scanning Electron Microscope. The cellulose monolith was cut into thin slice with $2-5 \mathrm{~mm}$ width. Several pretreatment was performed involving acetone treatment for $5 \mathrm{~h}$ and hexane treatment for $48 \mathrm{~h}$. The sample was air dried and oven dried at $50^{\circ} \mathrm{C}$. Porosity was calculated by the equation.

M1 and M2 refer to the total mass before and after drying, and and $\mathrm{V}$ indicate density of solution and total volume of the monolith, respectively.

Functionalization characterization: The pseudo-affinity sulfate content and anion-exchange nitrogen content were measured using Element Analyzer (CHNS/O 2400 Analyzer, PerkinElmer, USA) after thoroughly washing and drying treatment.

\section{Results and Discussion}

\section{Structure of the cellulose fiber monolith}

Silicon and organic polymer are the two major types of widely used monolith. Different approaches have been developed for making silicon and polymer monolith with wide pore size distribution. Pore size structure and distribution are key properties of the monolith and these predominately determine the separation range of the target molecule.

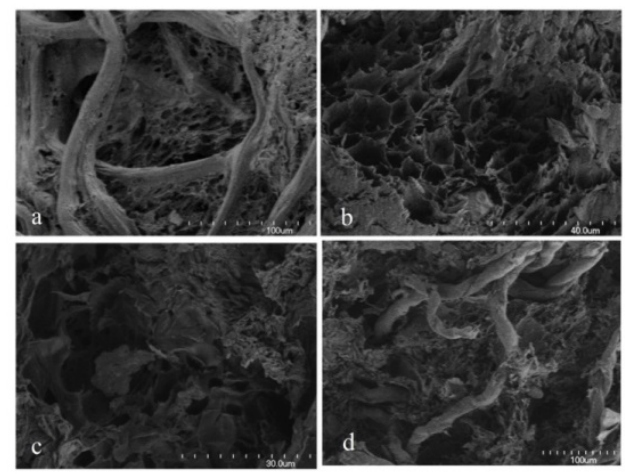

Figure 2: SEM image of cellulose fiber monolith.

For large particles such as virus with diameter larger than $100 \mathrm{~nm}$, the pore size of monolith should be large enough to allow the suspension to flow through. Kramberger et al. have demonstrated a commercial short monolith column for fast tomato mosaic virus separation [26]. However, the preparation of cellulose monolith was rarely studied and reported regarding controllability of the pore structure, and its mechanical properties. One of the difficulties for making cellulose monolith is to control pore size. According to the procedure of preparing cellulose membrane, the pore size and its distribution are primarily controlled by the cellulose concentration and quick exchange of the dissolving solvent with other organic solvent. Currently, the commercial cellulose membrane (without acetated or nitrated modification) with diameter ranging from 0.1 to $5 \mu \mathrm{m}$ could not satisfy the separation requirement for cell debris.

Methods to control pore sizes of cellulose membranes could not be readily applied for cellulose monolith. The monolith is much thicker than the membrane. As a result, conventional solvent evaporation and exchange methods will not be sufficient to generate and solidify pores inside the monolith. To control the pore size of the cellulose monolith, a porogen, such as calcium carbonate, was applied to the process. Several calcium carbonate nanoparticles with different diameters were available for generating pores. The particles chosen in our experiment were $10 \mu \mathrm{m}$ in diameter, based on the size of the separation target. Influenza virus and virus like particle (VLP) are typical hundreds of nanometers in diameter, ranging from $100-500 \mathrm{~nm}$, and the cell debris particle are up to several microns. During the preparation process, calcium carbonate was dispersed in cellulose dissolving solution with DEAE-cellulose serving as dispersing agent, and it was thoroughly mixed with cellulose fiber.

The amount of calcium carbonate added into the system partially determined the porosity of the monolith. The removal of calcium carbonate particle was facilitated by $0.35 \%$ of hydrochloride acid and stirring under vacuum. The concentration of hydrochloride acid had to remain low to avoid hydrolysis of the cellulose. During the pore generation process, calcium carbonate was first introduced into the $\mathrm{ED} / \mathrm{KSCN}$ solution. However, the calcium carbonate can't stay dispersed during the process due to lack of the dispersant. DEAEcellulose was added into the system to disperse the calcium carbonate. After the calcium carbonate was dissolved and washed, the DEAEcellulose would be more on the surface rather than inside the matrix. Pore size of the cellulose monolith can be controlled by calcium carbonate with different size.

In Figure 2 the pore size and structure suggest the successful application of calcium carbonate in generating pores inside the monolith. The pore size as indicated in the figure is $10 \mu \mathrm{m}$, which coincides with the calcium carbonate particle diameter. The pore structure of monolith was attributed to two different sources. One was the existence of porogen, calcium carbonate, which generated extensive pores inside monolith with a diameter of $10 \mu \mathrm{m}$. Another source for generating porous structure is the interaction of fibers with each other. As it can be seen in Figures $2 \mathrm{~d}$ and 3, the gap between fibers also contributes to the porous structure of the monolith ranging from $10 \mu \mathrm{m}$ to $30 \mu \mathrm{m}$.

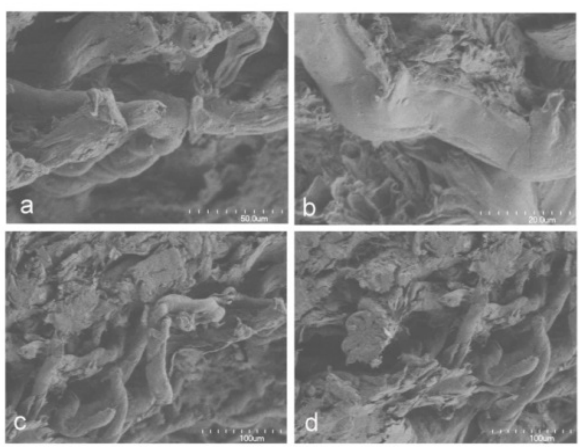

Figure 3: SEM image of microfiber inside the cellulose monolith.

To increase the mechanical property of the cellulose and ease of shaping the monolith into cylindrical process, we started the cellulose preparation process as follows: first, we employ Lyocell cellulose fiber instead of cellulose powder as a cellulose source. The advantage will be shown in the results section that deals with mechanical property. Secondly, the cellulose fiber was partially dissolved in the solution. Instead of totally dissolving the cellulose powder into the solution, the cellulose fiber went through mixing process together with calcium 
carbonate/DEAE-cellulose. The mixture was viscous and can be shaped into cylindrical mold for further processing. During the process, the cellulose fiber was partially dissolved and interacted with surrounding fiber to form interconnected matrix. The dissolving solution was then exchanged by organic solvent to solidify the monolith.

\section{Mechanical properties of the monolith}

Mechanical properties are important to characterize a monolith, especially for the ones intended for use under high pressure. Mechanical property reflects the inner-structure of the matrix material. High-density monolith material with small pore size generally has high Young's Modulus. However, for a highly porous material with relatively large pore size, retaining high mechanical property is a challenge. Only a few methods have been reported for enhancing mechanical property of highly porous material such as crosslinking and double networking $[27,28]$. However, the crosslinking method requires the functional groups to bind inside the monolith, which decreases the modification possibility and ligand accessibility.

\begin{tabular}{|l|l|l|l|l|}
\hline Sample & Lyocell fiber & DEAE fiber & Porosity & Young modulus(E MP) \\
\hline 1 & $54.0 \%$ & $46.0 \%$ & $86.1 \%$ & $0.144 \pm 0.0152$ \\
\hline 2 & $79.0 \%$ & $21.0 \%$ & $84.9 \%$ & $0.257 \pm 0.0520$ \\
\hline 3 & $99.5 \%$ & $0.05 \%$ & $85.8 \%$ & $0.315 \pm 0.0757$ \\
\hline
\end{tabular}

Table 1: Cellulose monolith composition and porosity (wt \%).

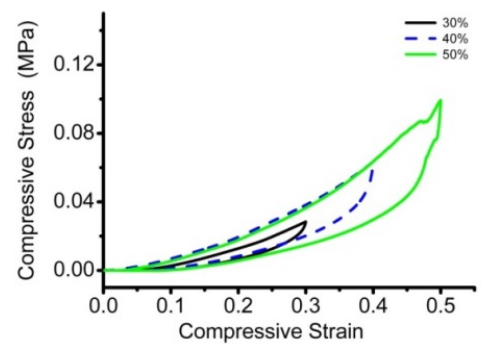

Figure 4: Compressive stain/stress measurement of cellulose monolith.

To examine the effect of Lyocell fiber on the mechanical property of the monolith, we tested the mechanical properties of the monolith by varying the ratio of Lyocell fiber to DEAE fiber as shown in Table 1. Mechanical properties were evaluated by $30 \%, 40 \%$ and $50 \%$ compression experiments and results are showed in Figure 4, which indicates that the compressive stress increases with higher compressive strain. The experiment result also indicated monolith can tolerant more than $50 \%$ compression without deformation. The results in Table 1 indicate that lower composition of Lyocell cellulose fiber will decrease the Young's Modulus. Young's Modulus was calculated at 30\% compression with a total height of $10 \mathrm{~mm}$. As seen in the Table 1, higher content of the cellulose fiber produced, higher Young's Modulus was achieved.

\section{Porosity of the cellulose monolith}

The superporous hydrogel has been prepared using organic polymer or silica as supporting material [29]. Commercial cellulose membrane has a pore size from $0.2 \mu \mathrm{m}$ to $5 \mu \mathrm{m}$ and porosity upto $90 \%$. Cellulose monolith prepared with calcium carbonate porogen has larger pore size than the current commercial cellulose membrane (without modification). Here, we have demonstrated that the porosity of cellulose fiber monolith could be controlled ranging up to $85 \%$. The porosity of monolith was controlled by the addition of the porogen, calcium carbonate. However, the decreased mechanical robustness was observed when the void volume of the monolith was increased. Hence, the mechanical property was enhanced by the fiber which blended into the monolith.

\section{Separation of influenza virus}

Recently, Optiz et al. proposed an economical pseudo-affinity sulfate-modified cellulose membrane device for influenza virus separation claiming simpler procedure, lower cost, and more stable modification over conventional affinity modifications [30].
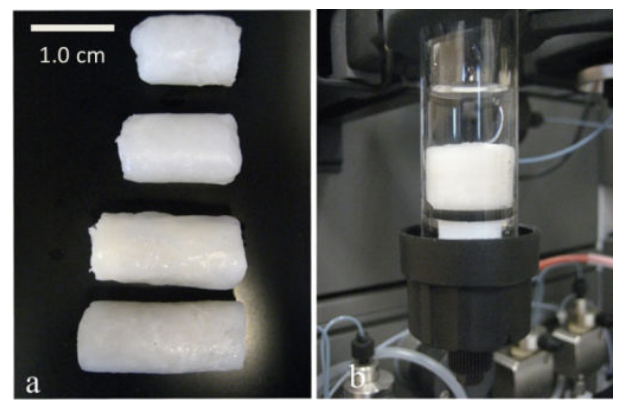

Figure 5: Image of cellulose fiber monolith (a): with $1.0 \mathrm{~cm}$ of diameter, and (b): cellulose monolith fitting in superloop for virus separation.

Sulfate modification could mimic the function of heparin, which could specifically attract surface protein-hemagglutinin on influenza virus. The efficiency of separation varied from $60 \%$ to $90 \%$ depending on different strains as demonstrated by the authors.

Anion-exchange modification has also been demonstrated for influenza virus separation, and the efficiency depends on strains and the host cells [2]. In this paper, two different separation modes were introduced sequentially and compared for the efficiency of the virus separation. According to the preparation procedure, the anion groups are primarily exposed on the pore surface after the calcium carbonate was dissolved and removed. When the sulfated cellulose fiber was flowed through the monolith, sulfated cellulose fiber with long chain would pack onto the upper surface of the cellulose monolith, and sulfated cellulose fiber with small chain would flow through the monolith and self-assemble onto the inner surface of the monolith. The sulfated cellulose attached onto the pore surface of the monolith due to the electrostatic interaction. Both the anionic modification and sulfated cellulose fiber will be primarily exposed to the virus containing media. The quantification of each functionalization was characterized by the Element Analyzer. The nitrogen content (stand for anion-exchange) was determined at 0.84 wt\% (duplicate 
measurement), and the sulfate content was measured at $0.89 \mathrm{wt} \%$ (duplicate measurement).

We performed the separation of influenza virus, H1N1(A/WSN/33), using both anion-exchange and pseudo-affinity modes. The cellulose monolith was fitted into the superloop as shown in Figure 5b. Figure 5a shows the monoliths with different proterties made by the procedures described earlier. The influenza virus separation was performed directly from the virus-containing MDCK cell culture without prior centrifugation. The separation efficiencies of virus, dsDNA, and protein are shown in Table 2. The results show that for the influenza virus, the pseudo-affinity cellulose have higher virus separation efficiency for virus, and less for dsDNA and protein contamination, which indicate sulfated functionalization is a better choice for this influenza virus.

\begin{tabular}{|l|l|l|l|}
\hline & Virus Retained & Protein Retained & dsDNA Retained \\
\hline Anion-exchange & $97.9 \% \pm 1.11 \%$ & $63.34 \% \pm 4.37 \%$ & $73.58 \% \pm 4.95 \%$ \\
\hline Pseudo-affinity & $99.7 \% \pm 0.02 \%$ & $39.55 \% \pm 3.25 \%$ & $20.67 \% \pm 2.69 \%$ \\
\hline
\end{tabular}

Table 2: Separation efficiency of biomolecules by two functionalization.

\section{Conclusion}

In this paper, we have demonstrated a new method to develop highly porous, mechanically robust cellulose monolith for separation of influenza virus. Two different types of functionalization have been introduced for influenza virus separation. The virus separation experiment showed that the newly developed monolith could be used for virus separation directly from the cell culture, and that pseudoaffinity functionalization has better virus separation efficiency, as well as less dsDNA and protein retention.

\section{Acknowledgement}

The authors are thankful to Dr. Juliusz Warzywoda of Texas Tech University for his assistance with the SEM images. We also thank the National Science Foundation for funding this work through a grant: CBET-0963017.

\section{References}

1. Finelli F, Fiore A, Dhara R, BrammerL, Shay DK, et al. (2008) Influenzaassociated pediatric mortality in the United States: Increase of staphylococcus aureus coinfection. Pediatrics 122: 805-811.

2. Wolff MW, Reichl U (2008) Downstream processing: from egg to cell culturederived influenza virus particles. Chem Eng Technol 31: 846-857.

3. Vicente T, Roldao A, Peixoto C, Carrondo MJT, Alves PM (2011) Largescale production and purification of VLP-based vaccines. J Invertebr Pathol 107: S42-S48.

4. Opitz L, Hohlweg J, Reichl U, Wolff MW (2009) Purification of cell culture-derived influenza virus A/Puerto Rico/8/34 by membrane-based immobilized metal affinity chromatography. J Virol Methods 161: 312-316.

5. Kalbfuss B, Wolff M, Morenweiser R, Reichl U (2006) Purification of cell culturederived human influenza $A$ virus by sizeexclusion and anionexchange chromatography. Biotechnol Bioeng 96: 932-944.

6. Nayak DP, Lehmann S, Reichl U (2005) Downstream processing of MDCK cell-derived equine influenza virus. J Chromatogr B 823: 75-81.
7. Sweet C, Stephen J, Smith H (1974) Purification of influenza viruses using disulphide-linked immunosorbents derived from rabbit antibody. Immunochemistry 11: 295-304.

8. Guiochon G (2007) Monolithic columns in high-performance liquid chromatography. J Chromatogr A 1168: 101-168.

9. Gusev I, Huang X, Horvath C (1999) Capillary columns with in situ formed porous monolithic packing for micro high-performance liquid chromatography and capillary electrochromatography J Chromatogr A 855: 273-290.

10. Svec F (2005) Recent developments in the field of monolithic stationary phases for capillary electrochromatography. J Sep Sci 28: 729-745.

11. Potter OG, Hilder EF (2008) Porous polymer monoliths for extraction: diverse applications and platforms. J Sep Sci 31: 1881-1906.

12. Sprob J, Sinz A (2011) Monolithic media for applications in affinity chromatography. J Sep Sci 34: 1958-1973.

13. Svec F, Tennikova TB (2003) Monolithic Materials: Preparation, Properties, and Applications.

14. Ruščić J, Gutiérrez-Aguirre I, Žnidarič MT, Kolundžija S, Slana A (2015) A new application of monolithic supports: The separation of viruses from one another. J Chromatogra A 1388: 69-78.

15. Venkatachalam ARK, Szyporta M, Kiener TK, Balraj P, Kwang J (2014) Concentration and purification of enterovirus 71 using a weak anionexchange monolithic column. Virol J 11: 99.

16. Oksanen HM, Domanska A, Bamford DH (2012) Monolithic ion exchange chromatographic methods for virus purification. Virology 434: 271-277.

17. Ghanem A, Ikegami T (2011) Recent advances in silicabased monoliths: Preparations, characterizations and applications. J Sep Sci 34: 1945-1957.

18. Siouffi AM (2003) Silica gel-based monoliths prepared by the sol-gel method: facts and figures. J Chromatogr A 1000: 801-818.

19. Namera A, Nakamoto A, Saito T, Miyazaki S (2011) Monolith as a new sample preparation material: Recent devices and applications. J Sep Sci 34: 901-924.

20. Mulder M (1996) Basic principles of membrane technology. Springer, Germany.

21. Ghosh R (2002) Protein separation using membrane chromatography: opportunities and challenges. J Chromatogr A 952: 13-27.

22. Zeng X, Ruckenstein E (2008) Membrane chromatography: Preparation and applications to protein separation. Biotechnol Progr 15: 1003-1019.

23. Schweiger RG (1972) Polysaccharide sulfates. I. cellulose sulfate with a high degree of substitution. Carbohyd Res 21: 219-228.

24. Chen G, Zhang B, Zhao J, Chen H (2013) Improved process for the production of cellulose sulfate using sulfuric acid/ethanol solution. Carbohyd Polym 95: 332-337.

25. Szretter KJ, Balish AL, Katz JM (2006) Current protocols in microbiology.

26. Kramberger P, Peterka M, Boben J, Ravnikar M, Štrancar A (2007) Short monolithic columns-A breakthrough in purification and fast quantification of tomato mosaic virus. J Chromatogra A 1144: 143-149.

27. Leventis N (2007) Three-dimensional core-shell superstructures: Mechanically strong aerogels. Accounts of Chemical Research 40: 874-884.

28. Leventis N, Mulik S, Wang X, Dass A, Patil VU, et al. (2008) Polymer nano-encapsulation of templated mesoporous silica monoliths with improved mechanical properties. J Non-Cryst. Solids 354: 632-644.

29. Chen J, Park H, Park K (1999) Synthesis of superporous hydrogels: Hydrogels with fast swelling and superabsorbent properties. J Biomed Mater Res 44: 53-62.

30. Opitz L, Lehmann S, Reichl U, Wolff MW (2009) Sulfated membrane adsorbers for economic pseudoaffinity capture of influenza virus particles. Biotech Bioeng 103: 1144-1154. 